\title{
INFLUENCIA DE LOS FACTORES DE RIESGO QUE PROVOCAN ACCIDENTES DE TRABAJO EN LA EMPRESA MINERA VICUS S.A.C. - BARRANCA
}

\author{
INFLUENCE OF THE RISK FACTORS THAT CAUSE WORK ACCIDENTS \\ IN THE MINING COMPANY VICUS S.A.C. - BARRANCA
}

Haydee Del Rosario Ramos Pachecoํ, Milagro Rosario Henríquez Suarez", Cesar Alexander López Ramos ${ }^{2}$, Héctor Serafín López Traslaviña ${ }^{2}$

\section{RESUMEN}

Objetivo: Identificar cuáles son los factores de riesgo que influyen en la incidencia de accidentes de trabajo. Materiales y Métodos: la investigación es aplicada, el diseño es de causa - efecto con el propósito de investigar la relación entre las variables que intervienen en el problema planteado, la población para la presente investigación la constituyen los funcionarios y trabajadores de la Empresa Minera "VICUS S.AC." que son 98, Resultados: Los factores de riesgo influyen en los accidentes de trabajo en la empresa minera VICUS S.A.C. EI $64 \%$ de los trabajadores afirman existen altos factores de riesgo los que inciden en accidentes de trabajo; pues desde una perspectiva empresarial, los accidentes alteran la producción, incrementando así los costos y, en ocasiones, poniendo en entredicho la reputación de la empresa. Conclusiones: Se recomienda a la Gerencia de la empresa minera VICUS S.A.C. y a los colaboradores que son primordial la cultura organizacional, la capacitación permanente y motivar a los trabajadores con la finalidad de prevenir accidentes laborales y así mejorar la calidad corporativa de la empresa.

Palabras claves: Empresa Minería; Factores de Riesgo; Accidentes de Trabajo.

\begin{abstract}
Objective: to identify which are the risk factors that influence the incidence of work accidents. Materials and Methods: the research is applied, the design is cause - effect with the purpose of investigating the relationship between the variables that intervene in the proposed problem, the population for the present investigation are the officials and workers of the Mining Company. VICUS S.AC. "which are 98, Results: Risk factors influence work accidents at the mining company VICUS SAC $64 \%$ of workers say there are high risk factors that affect work accidents; because from a business perspective, accidents alter production, thus increasing costs and, at times, putting into question the reputation of the company. Conclusions: The Management of the mining company VICUS S.A.C. and to the collaborators that are fundamental organizational culture, permanent training and motivate workers in order to prevent accidents at work and thus improve the corporate quality of the company.
\end{abstract}

Key words: Mining Company; Risk factor's; Work accidents.

\footnotetext{
${ }^{1}$ Docente. Facultad de Ciencias Sociales. Universidad Nacional José Faustino Sánchez Carrión. Lima - Perú. Email:haydeebshuacho@hotmail.com

${ }^{2}$ Administrativo. Universidad Nacional José Faustino Sánchez Carrión
} 


\section{INTRODUCCIÓN}

En la actualidad los procesos de globalización generan un gran impacto en la educación superior, pues cambian la manera de producir conocimiento, su aplicación y difusión. Las universidades como instituciones líderes de la educación superior y la promoción de la investigación cumplen un papel crucial en la gestión del servicio educativo y el conocimiento para afrontar los grandes desafíos que conlleva la globalización. De ahí la necesidad de adaptar sus roles y desarrollaren sus estudiantes competencias que les permitan anticiparse y responder a los cambios en los ámbitos local, regional e internacional. Los estudiantes aspiran hoy a carreras que les permitan emplearse rápidamente.

La globalización de la educación superior, según Deepak Nayyar (2012), conlleva efectos significativos para las comunidades locales y los países: ha tomado las universidades, ha incrementado sustancialmente la movilidad académica, ha expandido las corporaciones trasnacionales y se ha convertido en un fenómeno de alcance mundial.

Según Campero (2008), hoy se exige a las instituciones educativas del nivel superior formar personas competentes, lo que hace que la formación deba apuntar al vínculo con el campo del desempeño profesional y la participación social, pues la sola actividad académica y la acumulación de conocimientos o de información no garantizan el dominio y la experticia en competencias profesionales, menos aún si esa formación continúa siendo de carácter asignaturista o disciplinar, aislada, sin un eje integrador que permita comprender la complejidad de interrelaciones y sistemas propios del ejercicio en la vida del profesional en su contexto.

\section{MATERIAL Y MÉTODO}

Se realizó encuesta la cual permitió recoger información en forma directa y fidedigna de los 98 trabajadores de la empresa minera VICUS S.AC. La cual fue una evaluación y a la vez una crítica, que consistió en inspeccionar la validez y confiabilidad de los datos para corregir los errores y omisiones de acuerdo a sus reglas fijas, se ordenó y tabuló los resultados de la encuesta aplicada a los 98 trabajadores de la empresa minera VICUS S.A.C. mostrando los resultados calculados, graficados e interpretados con sus frecuencias y porcentajes. Se ha empleado la estadística no paramétrica mediante la prueba Chi-cuadrado de Pearson que se utiliza para probar la independencia de dos variables cualitativas entre sí, mediante la representación de los datos en tablas de contingencia o tabla de dos dimensiones.

\section{RESULTADOS}

Se presentan los resultados de la investigación, sobre la base de los objetivos y las variables de estudio lo que va a permitir la contrastación de las hipótesis y el alcance de los propósitos del estudio. Los resultados obtenidos según la encuesta de las categorías estudiadas de los Factores de Riesgo que provocan Accidentes de Trabajo en la Minera VICUS S.A.C. - Barranca se describen a continuación en la contrastación de la Hipótesis:

Hipótesis General HO: Los factores de riesgo no influyen en los accidentes de trabajo en la Empresa Minera VICUS S.A.C.

H1: Los factores de riesgo influyen en los accidentes de trabajo en la Empresa Minera VICUSS.A.C.

Tabla 1. Frecuencias Esperadas de los Factores de Riesgo y los Accidentes de Trabajo en la Empresa Minera VICUS S.A.C.

\begin{tabular}{cccc}
\hline $\begin{array}{c}\text { FACTORES DE } \\
\text { RIESGO }\end{array}$ & \multicolumn{2}{c}{ ACCIDENTES DE TRABAJO } & TOTAL \\
\hline INCIDE & NO INCIDE & \\
\hline BAJO & 57.6 & 10.4 & 68.0 \\
TOTAL & 25.4 & 4.6 & 30.0 \\
& 83.0 & 15.0 & 98.0 \\
\hline
\end{tabular}

Tabla 2. Prueba Chi - Cuadrado

\begin{tabular}{lccc}
\hline & Valor & Grado de libertad & Significación \\
\hline Chi-cuadrado & 10.839 & 1 & 0.001 \\
$\mathrm{~N}^{0}$ de casos & 98 & & \\
\hline
\end{tabular}

Interpretación: En el análisis estadístico se encontró que el valor de $p=0.001$ es menor que 0.05 , lo cual indica que se rechaza la hipótesis nula; es decir, queda demostrado que los factores de riesgo influyen en los accidentes de trabajo en la Empresa Minera VICUS S.A.C. Barranca

Hipótesis Específica 1

HO: La actitud de los funcionarios influye negativamente en el desempeño laboral de los trabajadores de la Empresa Minera VICUS S.A.C.

H1: La actitud de los funcionarios influye positivamente en el desempeño laboral de los trabajadores de la Empresa Minera VICUS S.A.C.

Tabla 3. Frecuencias Esperadas de las Actitudes de los Funcionarios en el desempeño laboral de los trabajadores de la Empresa Minera VICUS S.A.C. Barranca.

\begin{tabular}{cccc}
\hline $\begin{array}{c}\text { ACTITUD DE LOS } \\
\text { FUNCIONARIOS }\end{array}$ & DEICIENTE & INEFICIENTE & \\
\hline ADECUADA & 59.8 & 9.2 & 69.0 \\
INADECUADA & 25.2 & 3.8 & 29.0 \\
TOTAL & 85.0 & 13.0 & 98.0 \\
\hline
\end{tabular}

Tabla 4. Prueba Chi - Cuadrado

\begin{tabular}{lccc}
\hline & Valor & Grado de libertad & Significación \\
\hline Chi-cuadrado & 4.232 & 1 & 0.040 \\
$\mathrm{~N}^{0}$ de casos & 98 & & \\
\hline
\end{tabular}

En el análisis estadístico se encontró que el valor de $p$ $=0.040$ es menor que 0.05 , lo cual 
es decir, queda demostrado que la actitud de los funcionarios influye positivamente en el desempeño laboral de los trabajadores de la Empresa Minera VICUS S.A.C.

\section{Hipótesis Específica 2}

HO: La capacitación permanente no influye en la prevención de tipos y secuelas de accidentes de trabajo en la Empresa Minera VICUS S.A.C.

H1: La capacitación permanente influye en la prevención de tipos y secuelas de accidentes de trabajo en la Empresa Minera VICUS S.A.C.

Tabla 5. Frecuencias Esperadas de la Capacitación Permanente y la Prevención de Tipos de Accidentes de Trabajo en la Empresa Minera VICUS S.A.C. Barranca.

\begin{tabular}{cccc}
\hline $\begin{array}{c}\text { CAPACITACIÓN } \\
\text { PERMANENTE }\end{array}$ & PREVENCION DE ACCIDENTES & TOTAL \\
& PREVIENE & NO PREVIENE & \\
\hline $\begin{array}{c}\text { MEJORA LAACT. } \\
\text { LABORAL }\end{array}$ & 66.6 & 8.4 & 75.0 \\
NO MEJORA LA & 20.4 & 2.6 & 23.0 \\
$\begin{array}{c}\text { ACT. LABORAL } \\
\text { TOTAL }\end{array}$ & 87.0 & 11.0 & \\
\hline
\end{tabular}

Tabla 6. Prueba Chi - Cuadrado

\begin{tabular}{lccc}
\hline & Valor & Grado de libertad & Significación \\
\hline Chi-cuadrado & 6.662 & 1 & 0.010 \\
$\mathrm{~N}^{0}$ de casos & 98 & & \\
\hline
\end{tabular}

En el análisis estadístico se encontró que el valor de $p$ $=0.010$ es menor que 0.05 , lo cual indica que se rechaza la hipótesis nula; es decir, queda demostrado que la capacitación permanente influye en la prevención de tipos y secuelas de accidentes de trabajo en la Empresa Minera VICUS S.A.C.

Hipótesis Específica 3

HO: La motivación no influye en la cultura organizacional mostrada por los trabajadores de la Empresa Minera VICUS S.A.C.

H1: La motivación influye en la cultura organizacional mostrada por los trabajadores de la Empresa Minera VICUS S.A.C.

Tabla 7. Frecuencias Esperadas de la Motivación y la Cultura Organizacional mostrada por los trabajadores de la Empresa Minera VICUS S.A.C. Barranca.

\begin{tabular}{cccc}
\hline \multirow{2}{*}{ MOTIVACIÓN } & \multicolumn{2}{c}{ CULTURA ORGANIZACIONAL } & \\
& ADECUADA & INADECUADA & TOTAL \\
\hline ADECUADA & 47.8 & 12.2 & 60.0 \\
INADECUADA & 30.2. & 7.8. & 38.0 \\
TOTAL & 78.0 & 20.0 & 98.0
\end{tabular}

Tabla 8. Prueba Chi - Cuadrado

\begin{tabular}{lccc}
\hline & Valor & Grado de libertad & Significación \\
\hline Chi-cuadrado & 7.279 & 1 & 0.007 \\
$\mathrm{~N}^{\circ}$ de casos & 98 & & \\
\hline
\end{tabular}

Interpretación: En el análisis estadístico se encontró que el valor de $p=0.007$ es menor que 0.05 , lo cual indica que se rechaza la hipótesis nula; es decir, queda demostrado que la motivación influye en la cultura organizacional mostrada por los trabajadores de la Empresa Minera VICUS S.A.C.

\section{DISCUSIÓN}

Los factores de riesgo influyen en los accidentes de trabajo en la empresa minera VICUS S.A.C. EI 64\% de los trabajadores afirman existen altos factores de riesgo los que inciden en accidentes de trabajo; pues desde una perspectiva empresarial, los accidentes alteran la producción, incrementando así los costos y, en ocasiones, poniendo en entredicho la reputación de la empresa.

La actitud de los funcionarios influye positivamente en el desempeño laboral de los trabajadores de la empresa minera VICUS S.A.C. El $64 \%$ de los trabajadores perciben una adecuada actitud de los funcionarios por lo que el desempeño laboral es eficiente; es conveniente promover la comunicación asertiva entre empresa y colaborador mediante programas.

La capacitación permanente influye en la prevención de tipos y secuelas de accidentes de trabajo en la empresa minera VICUS S.A.C. El $71 \%$ de los trabajadores reciben capacitación permanente, lo que mejora su actividad laboral a la vez previenen accidentes laborales. En el ámbito individual, los costos personales de un accidente, tanto emocionales como económicos, pueden ser elevados. por lo que se debe disminuir los accidentes de trabajo.

La motivación influye en la cultura organizacional mostrada por los trabajadores de la empresa minera VICUS S.A.C. El 54\% de los trabajadores tienen una adecuada motivación laboral y cultura organizacional.

Dentro de este contexto se recomienda que:

Se debe aplicar el principio de prevención donde el empleador garantice, en el centro de trabajo el establecimiento de los medios y condiciones que protejan la vida, la salud y el bienestar de los trabajadores.

Se debe practicar el principio de la responsabilidad donde el empleador asumirá las implicancias económicas, legales y de cualquier otra índole, como consecuencia de un accidente o enfermedad que sufra el trabajador en el desempeño de sus funciones o consecuencias de él, conforme a las normas vigentes.

Aplicar el sistema de gestión de seguridad y salud en el trabajo y desarrollar programas de sensibilización tanto para los colaboradores y empleadores para disminuir los índices de accidentes de trabajo

Promover la comunicación asertiva entre empresa y colaborador mediante programas.

\section{AGRADECIMIENTO}

Se agradece a la empresa minera VICUS S.A.C. por brindar las facilidades para poder realizar la investigación y poder tomar en consideración las recomendaciones para el bien de ella misma. 


\section{REFERENCIAS BIBLIOGRÁFICA}

Acero Rosales, Tomas Manuel (2004) "Costos por accidentes de trabajo en la Minería Peruana entre los años 1994 y 1998"

Texto Único Ordenado de la Ley General de Minería D.S. N ${ }^{\circ}$ 014-92-EM, del 02 de Junio de 1992.

Ministerio De Energía Y Minas - Dirección de Fiscalización Minera Estadística de Accidentes en la Minería - 1994

Ministerio De Energía Y Minas - Dirección de Fiscalización Minera -Estadística de Accidentes en la Minería - 1995.

Ministerio De Energía Y Minas - Dirección de Fiscalización Minera - Estadística de Accidentes en la Minería-1996.

Ministerio De Energía Y Minas - Dirección de Fiscalización Minera - Estadística de Accidentes en la Minería - 1997.

Ministerio De Energía Y Minas - Dirección de Fiscalización Minera -Estadística de Accidentes en la Minería -1998.

Publicación PERÚ MINERO №7, Marzo de 1997, pág. 23 a 25.

Revista del Consejo Superior de Minería, "MINERÍAN 245 1997, Ing. Jorge DiazArtieta - "Filosofía de la Seguridad Ocupacional en laActividad Minera"

Hienrich, H. W. - Prevención de Accidentes Industriales, Asociación Mexicana de Higiene y Seguridad, 1978.

Oficina Internacional del Trabajo OIT.- La Prevención de Accidentes - Manual de Educación para Trabajadores, 1982. Reglamento de Seguridad e Higiene Minera D.S. $\mathrm{N}^{\circ}$ 023-92-EM, 09-10-92

Cano Mujica, Diana Delia (2009) "Características epidemiológicas de accidentes de trabajo atendidos en el Hospital Regional de Arequipa". Universidad Católica de Santa maría.

Alonso Olea, MANUEL (2010) "Derecho del trabajo y de la Seguridad Social” Editorial Civitas. Edición 26 Madrid.

Blake, Roland P. - Seguridad Industrial, Edit. Diana Lib. Studium S.A., Método Simonds, pág. 54-59, 1979.

Anuario Minero 1997- Dirección General De Minería Ministerio De Energía Y Minas - Sub Sector Minería.

Alonso Olea. Manuel; Derecho del Trabajo y de la Seguridad Social - Editorial Civitas Ediciones. 26a Ed. - Madrid.

Arias Galicia Femando; Administración de los recursos humanos: para el alto desempeño: Editorial Trillas. Sexta edición: 2006: México, D.F.

Cabanellas "Derecho de los Riesgos de Trabajo" - Editorial Omeba - Buenos Aires Argentina.

Chiavenato Idalberto; Administración de recursos humanos; Editorial Me Graw Hill, Octava edición; 2007; México, D.F.
Danatro Daniel; Ausentismo laboral de causa médica en una institución; Editorial Montevideo, primera edición; 1994.

Díez Millán Enrique; Millán Bartolomé José - La Jomada Laboral, Problemática Legal y Práctica - Fundación Confe-Metal Editorial - España

Gestol C. Cereijo; Ausentismo laboral: Editorial Interamericana. Primera Edición; México 1995.

Gomez Valdez. Francisco Derecho Del Trabajo: Relaciones Individuales de Trabajo; 1ra. Edición; Lima Perú, 1996.

Hellriegel, Don; Administración: Un enfoque basado en Competencias; Editorial Thomson, Edición 10; 2005

Ladou, Joseph Medicina Laboral y Ambiental; segunda edición traducida al español. Editorial el Manual Moderno, México D.F. 1999.

Lettieri, Antonio. "La Revolución en el Tiempo de Trabajo" Revista de Derecho Social N ${ }^{\circ} 7$, Editorial Bomarzo Albacete 1999.

Molina N. Cristóbal y otros, Manual de Derecho del Trabajo Editorial Gomares. Sexta Edición 10/2008 Editorial Lex Nova - Madrid 2008.

Monereo P. Luis y otros "Tratado Práctico a la Legislación Reguladora de los Accidentes de Trabajo y Enfermedades Profesionales" - Universidad de Granada - 2006

Ramirez, Luis Enrique "Riesgos del Trabajo" - Manual Práctico. 4a Ed. Editorial Montevideo - Buenos Aires Argentina - 2007

Rendon V. Jorge y otros "Flexibilidad o Derechos Sociales" Trabajo y Seguridad Social - Universidad Nacional Mayor de San Marcos - Edial Lima 2001.

Roger R. Rosa y Colligan Michael J. El trabajo por turnos en lenguaje sencillo. Traducido al español Julio 1997. $\mathrm{NIOSH}$.

Jurisprudencia y Doctrina Constitucional Laboral - Tribunal Constitucional del Perú - Centro de Estudios Constitucionales - Lima - 2006

Revista Filosofía del Control de Riesgos - Consejo Interamericano de Seguridad - EEUU. 1994

Tesis de Maestría en Salud Ocupacional "Costos por accidentes de trabajo en la minería peruana (1994 1998), de Tomás M. Acero Rosales. Año 2004.

"El trabajo peligroso mata a millones y cuesta billones"; 26 de Mayo 2003 